\title{
Mott-Schottky behavior of strongly pinned double Schottky barriers and characterization of ceramic varistors
}

\author{
D. Fernández-Hevia and J. de Frutos ${ }^{a}$ \\ Escuela Técnica Superior de Ingenieros de Telecomunicación, Universidad Politécnica de Madrid, \\ Ciudad Universitaria s/n, 28040 Madrid, Spain
}

\author{
A. C. Caballero and J. F. Fernández ${ }^{\text {b) }}$ \\ Departamento de Electrocerámica, Instituto de Cerámica y Vidrio, Consejo Superior de Investigaciones \\ Cientificas, 28500 Arganda del Rey, Madrid, Spain
}

(Received 7 January 2002; accepted for publication 10 June 2002)

\begin{abstract}
This work studies the capacitive behavior of an intergranular double Schottky barrier, which describes nonlinear charge transport in polycrystalline semiconductors. It is found that: (i) a widely applied version of the Mott-Schottky equation can be inadequate, and can lead to significant errors; (ii) a property called strong barrier pinning (SBP), underlies most attempts to obtain physical parameters from $C-V$ measurements; and (iii) under SBP, known results from one-sided Schottky barriers can be used to analyze $C-V$ response, showing that correct physical parameters are obtained at low frequency and that high frequency measurements are not advantageous. A new characterization method is introduced, which allows high voltage devices to be directly measured, and yields comparative information about average donor density and barrier height. Besides its technological applicability, the method simplifies the study of scale effects. Experimental results support the theoretical considerations and the proposed characterization method. The usefulness of the method is illustrated by studying the effects of current-pulse degradation on the physical parameters of high voltage varistors. (C) 2002 American Institute of Physics.
\end{abstract}

[DOI: $10.1063 / 1.1498968]$

\section{INTRODUCTION}

In polycrystalline semiconductors, nonlinear charge transport is governed by the grain boundary (GB) electronic structure, ${ }^{1,2}$ and described through a double Schottky barrier (DSB) model. ${ }^{3-5}$ Capacitance versus voltage measurements are widely used to characterize these materials, ${ }^{6-10}$ employing either the "standard" one-sided Mott-Schottky equation (stemming from one-sided Schottky barrier theory ${ }^{11}$ ), or a modified version ${ }^{12}$ that takes into account the double-sided nature of the barrier. Physical parameters, such as barrier height and donor density, have been extracted from these measurements. However, an unsatisfactory situation arises, because the very existence of Mott-Schottky behavior (in the sense of a linear relation between applied voltage and inverse-squared parallel capacitance) is not clearly established and relies upon tacit or undefined assumptions. A general unawareness seems to exist of the experimental conditions leading to Mott-Schottky behavior, and it appears that a bridge between general GB admittance theory ${ }^{4,5}$ and specific $C-V$ characterization procedures is lacking. Under these circumstances, physical insight is lost, incorrect conclusions can be inferred from experiment, and complex measurement systems are used to extract information that could be obtained by simpler methods. Therefore, it is necessary to clarify what kind of information is possible to extract from

${ }^{a)}$ Electronic mail: jfrutos@ fis.upm.es

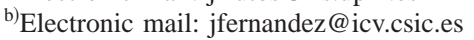

$C-V$ response, depending on the various experimental conditions, and how this information relates to Mott-Schottky behavior and GB admittance theory. ${ }^{4,5}$

The purpose of this work is to identify the conditions that lead to pure Mott-Schottky behavior, and to other meaningful $C-V$ characterization procedures, providing interpretation criteria that allow useful information to be extracted. The cases without and with deep donors are separately studied. We then propose a new $C-V$ measurement method, which exploits the aforementioned conditions. Its usefulness is illustrated through the study of the effects of current-pulse degradation on the physical parameters of high voltage ceramic varistors.

\section{DOUBLE SCHOTTKY BARRIER MODEL}

The arguments and methods proposed below draw heavily upon the DSB model approximations and equations for the barrier geometry. Hence, we summarize the form and origin of these expressions, simultaneously settling the notation. Figure 1 depicts a DSB, typical of ceramic varistors $^{2}$ and other semiconductor compounds. $E_{C}(x, V)$ $=-e \times \Phi(x, V)$ is the geometry of the conduction band under a dc bias $V=V_{\mathrm{dc}}, \Phi(x, V)$ is the associated electrostatic potential and $\Phi_{\mathrm{B}}(V) \equiv-\left.\Phi(x, V)\right|_{x=0}$ is the barrier height. The shallow donor, with density $N_{0}=n \equiv$ bulk free electron density and energy $E_{0}(x, V)$, is everywhere ionized. Deep lying donors (densities $N_{\alpha}$, energies $E_{\alpha}(x, V), \alpha=1, \ldots, d$ numbered from shallower to deeper), are occupied (neutral) in the bulk. The DSB is assumed homogeneous, so these 


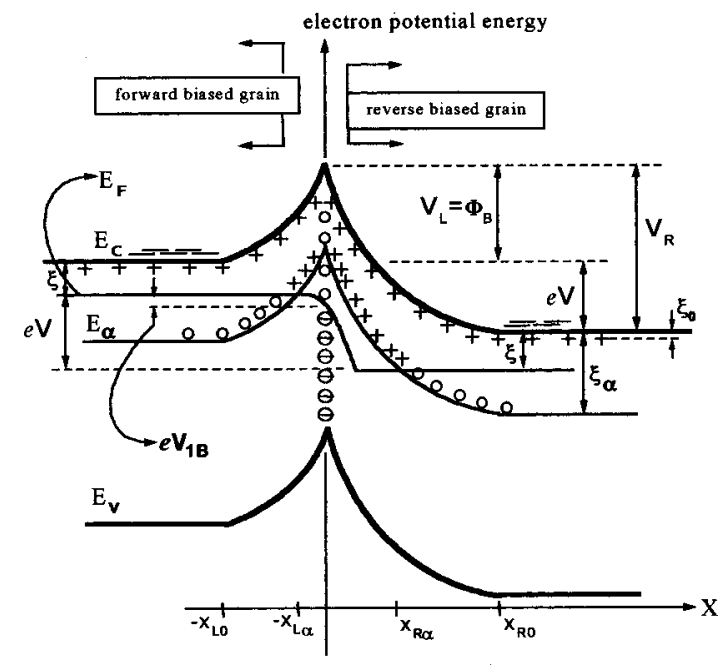

FIG. 1. Schematic energy band diagram of a double Schottky barrier, describing a grain boundary in a polycrystalline semiconductor. Open circles are neutral states. The quantity $e V_{1}$ is the energy difference between the bulk Fermi level in the forward-biased grain and the quasi-Fermi level at the boundary.

defect densities are constant throughout. The conduction band and the defect energies bend in parallel, so the energy separation $\xi_{\alpha} \equiv E_{C}(x, V)-E_{\alpha}(x, V) \quad(\alpha=0, \ldots, d) \quad$ remains constant everywhere for any bias. A surface density of states $N_{S}(E)$ exists at the GB, so that $N_{S}(E) d E$ is the number of available energy levels between $E$ and $E+d E$ by unit GB area. Free carriers are assumed to be negligible within the well defined limits $x=-x_{\mathrm{L} 0}$ and $x=x_{\mathrm{R} 0}$ (see Fig. 1) of the space charge (depletion) region, $5,13,14$ where neutrality is maintained be-

$$
\Phi(x, V)=\left\{\begin{array}{ll}
\frac{-e}{2 \varepsilon_{0} \varepsilon} \sum_{\alpha=0}^{d} N_{\alpha} \theta\left(x+x_{\mathrm{L} a}\right)\left(x+x_{\mathrm{L} \alpha}\right)^{2} & -x_{\mathrm{L} 0} \leqslant x \leqslant 0 \\
V-\frac{e}{2 \varepsilon_{0} \varepsilon_{r}} \sum_{\alpha=0}^{d} N_{\alpha}\left[1-\theta\left(x-x_{\mathrm{R} \alpha}\right)\right]\left(x-x_{\mathrm{R} \alpha}\right)^{2} & 0 \leqslant x \leqslant x_{\mathrm{R} 0}
\end{array},\right.
$$

where $\varepsilon_{0}, \varepsilon_{r}$ have the usual meaning, and the energy zero is chosen at the bottom of the forward-biased conduction band, which remains constant because the voltage fully drops in the reverse biased depletion region. ${ }^{3}$ The set of equations that determine the $2 d+3$ unknowns $x_{\mathrm{L} \alpha}, x_{\mathrm{R} \alpha}$ and $\Phi_{B}(\alpha$ $=0, . ., d)$ were written and solved for the first time by Blatter and Greuter, ${ }^{5}$ with a resulting barrier height:

$$
\begin{aligned}
& \Phi_{\mathrm{B}}\left(V, Q_{\mathrm{S}}\right) \approx U_{\mathrm{R}}\left(1-\frac{V}{4 U_{\mathrm{R}}}\right)^{2} \\
& U_{\mathrm{R}}(V) \equiv \frac{Q_{\mathrm{S}}(V)^{2}}{8 e \varepsilon_{0} \varepsilon_{r} N_{\mathrm{D}}} ; \quad N_{D} \equiv \sum_{\alpha=0}^{d} N_{\alpha} \\
& \left.\Phi_{\mathrm{B}}^{(0)} \equiv \Phi_{\mathrm{B}}\left(V, Q_{\mathrm{S}}\right)\right|_{V=0} \approx \frac{\left(Q_{S}^{(0)}\right)^{2}}{8 e \varepsilon_{0} \varepsilon_{r} N_{D}},
\end{aligned}
$$

tween the positive screening charge due to ionized donors and the excess negative charge trapped in the GB; away from this region into the bulk, the positive charge due to the shallow donor is balanced by electrons in the conduction band. Deep acceptor defects can be incorporated in the model by subtracting their density from that of the ionized shallow defects, and treating them as donors. ${ }^{5}$ The occupation statistics of all these states, can be described by a quasi-Fermi level $^{15} E_{\mathrm{F}}(x, V)$ :

$$
\begin{aligned}
& E_{\mathrm{F}}(x, V) \\
& \quad= \begin{cases}-\xi, & x \leqslant-x_{\Gamma-}\left(x_{\Gamma-}>0, x_{\Gamma-} \approx 0\right), \\
-\xi-e V_{1}(x, V), & -x_{\Gamma-} \leqslant x \leqslant x_{\Gamma+}, \\
-\xi-e V, & x_{\Gamma+} \leqslant x\left(x_{\Gamma+} \approx x_{\mathrm{R} 0}-x_{\mathrm{L} 0}\right),\end{cases}
\end{aligned}
$$

where $\xi$ is the energy separation between the conduction band and the Fermi level in the bulk. Near the GB, the quasiFermi level bends down in parallel ${ }^{15}$ with $E_{C}$ (driven by the function $V_{1}$ ), in such a way that the crossing points between $E_{\alpha}(x, V)$ and $E_{\mathrm{F}}(x, V)$, denoted $-x_{\mathrm{L} \alpha}$ and $x_{\mathrm{R} \alpha}(\alpha=1, . ., d)$, always lie in the flat regions of $E_{F}\left(x<-x_{\Gamma-}\right.$ and $\left.x>x_{\Gamma+}\right)$. The DSB geometry is determined by solving the Poisson equation for $\Phi(x, V)$ in $-x_{\mathrm{L} 0} \leqslant x \leqslant x_{\mathrm{R} 0}$, with boundary conditions $\Phi(-\infty)=\Phi\left(-x_{\mathrm{L} 0}\right)=0$ and $\Phi(+\infty)=\Phi\left(x_{\mathrm{R} 0}\right)=V$ $(>0)$; the charge density is nonhomogeneous, due to the spatial variation of $E_{\alpha}$ and $E_{\mathrm{F}}$ in the Fermi distribution $f_{\mathrm{F}}\left(E_{\alpha}, E_{\mathrm{F}}\right)$. The Schottky approximation ${ }^{5,13,14}$ is assumed, hence turning the charge density into a sum of homogeneous regions $\rho(x)=-e \sum_{\alpha=0}^{d} N_{\alpha}\left[\theta\left(x+x_{\mathrm{L} \alpha}\right)-\theta\left(x-x_{\mathrm{R} \alpha}\right)\right]$, and the solution of Poisson equation into a sum of parabolas: ${ }^{1,4}$

where the superscript "(0)" means zero-bias values and $Q_{S}(V)$ is the GB trapped charge by unit area, determined by the interface quasi-Fermi level. In steady state, and within the thermionic emission model, $V_{1}$ can be evaluated at the GB as ${ }^{3,15}$

$$
\begin{aligned}
e V_{1 \mathrm{~B}}\left(V_{\mathrm{dc}}\right) & \left.\equiv e V_{1}\left(x, V_{\mathrm{dc}}\right)\right|_{x=0} \\
& =k_{\mathrm{B}} T \ln \left[2 /\left(1+e^{-e V_{\mathrm{dc}} / k_{\mathrm{B}} T}\right)\right],
\end{aligned}
$$

so the interface quasi-Fermi level is $\left.E_{\mathrm{FB}}(V) \equiv E_{\mathrm{F}}(x, V)\right|_{x=0}$ $=-\xi-e V_{1 \mathrm{~B}}(V)$, and $Q_{\mathrm{S}}$ is given by

$$
\begin{aligned}
-Q_{S} & \equiv-Q_{\mathrm{S}}\left[V ; N_{\mathrm{S}}(E)\right] \\
& =e \int_{E_{V}}^{E_{C}} N_{\mathrm{S}}(E)\left[f_{\mathrm{F}}\left(E, E_{\mathrm{FB}}\right)-f_{\mathrm{F}}\left(E, E_{\mathrm{FB} b}\right)\right] d E,
\end{aligned}
$$


where $E_{\mathrm{FB}_{b}}$ is a fictitious Fermi level describing the neutral interface. $^{3}$

\section{GRAIN BOUNDARY CAPACITANCE WITHOUT DEEP DONORS}

\section{A. General $C-V$ behavior and the strong barrier pinning property}

Some materials present very small deep donor contribution: grain boundaries in silicon ${ }^{16}$ and $\mathrm{GaAs}^{17}$ have been characterized without taking deep donors into account. If only shallow donors are considered, Eq. (2) is notoriously simplified to

$\Phi(x, V)= \begin{cases}-\left(e N_{0} / 2 \varepsilon_{0} \varepsilon_{r}\right)\left(x+x_{\mathrm{L} 0}\right)^{2}, & -x_{\mathrm{L} 0} \leqslant x \leqslant 0, \\ -\left(e N_{0} / 2 \varepsilon_{0} \varepsilon_{r}\right)\left(x-x_{\mathrm{R} 0}\right)^{2}+V, & 0 \leqslant x \leqslant x_{\mathrm{R} 0} .\end{cases}$

Continuity of the potential at the GB (see Fig. 1) implies

$$
\begin{aligned}
-\Phi_{\mathrm{B}} \equiv & \Phi\left(0^{-}\right)=\Phi\left(0^{+}\right) \Rightarrow V=\left(e N_{0} x_{\mathrm{R} 0}^{2} / 2 \varepsilon_{0} \varepsilon_{r}\right) \\
& -\left(e N_{0} x_{\mathrm{L} 0}^{2} / 2 \varepsilon_{0} \varepsilon_{r}\right) \equiv V_{\mathrm{R}}-V_{\mathrm{L}} \equiv V_{\mathrm{R}}-\Phi_{\mathrm{B}},
\end{aligned}
$$

which yields a simple relation between the edges of the depletion region, the barrier height, and the applied voltage that by no means is true in the general (non-negligible deep donors) case:

$$
\begin{aligned}
& x_{\mathrm{L} 0}\left[V, \Phi_{\mathrm{B}}(V)\right]=\sqrt{2 \varepsilon_{0} \varepsilon_{r} \Phi_{\mathrm{B}} / e N_{0}} \\
& x_{\mathrm{R} 0}\left[V, \Phi_{\mathrm{B}}(V)\right]=\sqrt{2 \varepsilon_{0} \varepsilon_{r}\left(\Phi_{\mathrm{B}}+V\right) / e N_{0}} .
\end{aligned}
$$

The barrier height is still given by Eqs. (3) and (4), changing $N_{\text {D }}$ into $N_{0}$. Using Eq. (9), we can write the geometrical capacitance by unit area of the forward and reverse biased sides of the GB as

$$
\begin{aligned}
& C_{\mathrm{L}}(V) \equiv \frac{\varepsilon_{0} \varepsilon_{r}}{x_{\mathrm{L} 0}(V)}=\sqrt{\frac{e \varepsilon_{0} \varepsilon_{r} N_{0}}{2 \Phi_{\mathrm{B}}}} \\
& C_{\mathrm{R}}(V) \equiv \frac{\varepsilon_{0} \varepsilon_{r}}{x_{\mathrm{R} 0}(V)}=\sqrt{\frac{e \varepsilon_{0} \varepsilon_{r} N_{0}}{2\left(\Phi_{\mathrm{B}}+V\right)}} .
\end{aligned}
$$

Defining the total series geometrical capacitance (by unit area) as $C_{\mathrm{S}} \equiv C_{\mathrm{L}} C_{\mathrm{R}} /\left(C_{\mathrm{L}}+C_{\mathrm{R}}\right)$, Eq. (10) yields

$$
\left(\frac{1}{C_{\mathrm{S}}}-\frac{1}{C_{\mathrm{L}}}\right)^{2}=\frac{2}{e \varepsilon_{0} \varepsilon_{r} N_{0}}\left(\Phi_{\mathrm{B}}+V\right),
$$

which is generally valid if we properly interpret $C_{\mathrm{L}}$ $\equiv C_{\mathrm{L}}\left(\Phi_{\mathrm{B}}\right)$ and $\Phi_{\mathrm{B}} \equiv \Phi_{\mathrm{B}}\left(V, Q_{\mathrm{S}}\right)$. Equation (11), as it stands, does not allow to obtain neither $\Phi_{\mathrm{B}}^{(0)}$ nor $N_{0}$ : the right-hand side of (11) is a complicated function of $V$ (by no means a straight line) and does not contain the equilibrium barrier height $\Phi_{\mathrm{B}}^{(0)}$ as a parameter. We can transform (11) into a useful expression by assuming strong barrier pinning (SBP) conditions:

$$
\begin{aligned}
& \exists V_{\Phi}: 0 \leqslant V \leqslant V_{\Phi} \Rightarrow \Phi_{\mathrm{B}} \approx \Phi_{\mathrm{B}}^{(0)} \equiv \text { const; } \\
& V_{\mathrm{L}}=\Phi_{\mathrm{B}} \cong \Phi_{\mathrm{B}}^{(0)} ; \quad V_{\mathrm{R}}=\Phi_{\mathrm{B}}+V \cong \Phi_{\mathrm{B}}^{(0)}+V,
\end{aligned}
$$

meaning that, as soon as voltage increases, the barrier tends to decrease, hence dragging additional portions of the interface density of states (which is fixed with respect to the conduction band) below the GB quasi-Fermi level, thus increasing the amount of trapped charge $Q_{S}$ and inhibiting the barrier decrease. It should be noted that $(12) \rightarrow x_{\mathrm{L} 0}$ $\approx$ constant. The SBP property, expressed by Eq. (12), seems to be firmly established for a class of technologically important materials. It traces back to the pioneering work of Taylor, Odell, and Fan, ${ }^{18}$ who used it in their model for GBs in germanium, finding a reasonable agreement with experimental results. Also, numerical models sustain the existence of a $\mathrm{SBP}$ regime ${ }^{5}$ and, for commercial $\mathrm{ZnO}$ ceramic varistors, direct barrier height measurements ${ }^{9}$ have given almost constant average barrier height for applied fields up to $1.5 \mathrm{~V} / \mathrm{GB}$.

Introducing (12) in (11) we obtain

$$
\begin{aligned}
& \left(\frac{1}{C_{\mathrm{S}}}-\frac{1}{2 C_{\mathrm{S}}^{(0)}}\right)^{2}=\frac{2}{e \varepsilon_{0} \varepsilon_{r} N_{0}}\left(\Phi_{\mathrm{B}}^{(0)}+V\right) \\
& \left.C_{S}^{(0)} \equiv C_{\mathrm{S}}(V)\right|_{V=0}=\frac{1}{2} \sqrt{\frac{e \varepsilon_{0} \varepsilon_{r} N_{0}}{2 \Phi_{\mathrm{B}}^{(0)}}},
\end{aligned}
$$

which is a modified Mott-Schottky equation. ${ }^{12}$ Here, the role of (12) as underlying assumption is uncovered: when Eq. (13) was originally deduced, ${ }^{12}$ it was only assumed that the full applied voltage drops through the reverse biased grain, which is an insufficient hypothesis because it does not imply a negligible $\Phi_{\mathrm{B}}$ variation. Condition (12) must be explicitly assumed in order to jump from (11) to (13).

\section{B. Capacitance of the grain boundary in strong barrier pinning regime}

The capacitance (by unit area) is calculated from the oscillating current densities that flow in response to a timedependent signal $\Delta V(t)$, added to the dc bias. The applied voltage is now given by $V(t)=V_{\mathrm{dc}}+\Delta V \equiv V_{\mathrm{dc}}+V_{0} e^{i \omega t}$, with $V_{0}$ usually satisfying the small signal condition $e V_{0} \ll k_{\mathrm{B}} T$. In order to establish the consequences of assumption (12), we begin by writing the small signal capacitance of the GB as $^{4}$

$$
\begin{aligned}
C(\omega)= & C_{\mathrm{S}}+\frac{C_{\mathrm{R}}}{C_{\mathrm{L}}+C_{\mathrm{R}}} \operatorname{Re}\left[C_{Q}(\omega)\right] \\
& -\frac{e J_{\mathrm{dc}}}{\omega k_{\mathrm{B}} T\left(C_{\mathrm{L}}+C_{\mathrm{R}}\right)} \operatorname{Im}\left[C_{Q}(\omega)\right] .
\end{aligned}
$$

Here, $\quad J_{\mathrm{dc}}=A \times \exp \left[-\left(e \Phi_{\mathrm{Bdc}}+\xi\right) / k_{\mathrm{B}} T\right] \times\left[1-\exp \left(-e V_{\mathrm{dc}} /\right.\right.$ $\left.\left.k_{\mathrm{B}} T\right)\right]$ is the thermionic current emitted over the barrier in steady state, $\Phi_{\mathrm{Bdc}} \equiv \Phi_{\mathrm{B}}\left(V_{\mathrm{dc}}, Q_{\mathrm{Sdc}}\right), Q_{\mathrm{Sdc}} \equiv Q_{\mathrm{S}}\left[V_{\mathrm{dc}}, N_{\mathrm{S}}(E)\right]$, and $C_{\mathrm{L}}, C_{\mathrm{R}}$, and $C_{\mathrm{S}}$ are evaluated at $V_{\mathrm{dc}}$. The complex quantity $C_{Q}(\omega)$, contains the details of the distribution and dynamic properties of GB states: ${ }^{4}$

$$
\begin{aligned}
& C_{Q}(\omega)=K I(i \omega) /\left[\left(C_{\mathrm{L}}+C_{\mathrm{R}}\right) k_{\mathrm{B}} T / e^{2}+I(i \omega)\right] \\
& K=C_{\mathrm{R}}-\frac{C_{\mathrm{L}}+C_{\mathrm{R}}}{1+e^{e V_{\mathrm{dc}} / k_{\mathrm{B}} T}} \\
& I(i \omega)=\int_{E_{V}}^{E_{C} N_{\mathrm{S}}(E) \times f_{\mathrm{F}}\left(E, E_{\mathrm{FBdc}}\right) \times\left[1-f_{F}\left(E, E_{\mathrm{FBdc}}\right)\right]} \frac{(1}{1+i \omega \tau f_{\mathrm{F}}\left(E, E_{\mathrm{FBdc}}\right)} d E \\
& \tau \equiv(e / 2 A \sigma) \times \exp \left[\left(e \Phi_{\mathrm{Bdc}}+\xi+e V_{1 \mathrm{Bdc}}\right) / k_{\mathrm{B}} T\right],
\end{aligned}
$$


where $\tau$ establishes the time scale for the barrier reaction to the measurement signal, $E_{\mathrm{FBdc}} \equiv E_{\mathrm{FB}}\left(V_{\mathrm{dc}}\right)=-\xi-e V_{1 \mathrm{Bdc}}$, $e V_{1 \mathrm{Bdc}} \equiv e V_{1 \mathrm{~B}}\left(V_{\mathrm{dc}}\right)$ is given by Eq. (5), $A \equiv A^{*} T^{2}$ ( $A^{*}$ is Richardson's constant), and $\sigma$ is the capture cross section of GB states. The capacitance $C_{\mathrm{S}}$, also denoted $C_{\mathrm{HF}}$, gives the high frequency limit of (14): $\lim I(i \omega)=\lim C_{Q}(\omega)$ $=0 \Rightarrow \lim C(\omega)=C_{\mathrm{S}}$.

Examining the original calculations performed by Pike, ${ }^{4}$ it can be seen that the first two terms in (14) come from the displacement current created by the oscillation of the shallow screening charge

$$
J_{\mathrm{D}}=\dot{Q}_{\mathrm{R}} \equiv \frac{d}{d t} \sqrt{2 e \varepsilon_{0} \varepsilon_{r} N_{0}\left(\Phi_{\mathrm{B}}+V\right)}=\frac{C_{\mathrm{R}}}{e}\left(\dot{\Phi}_{\mathrm{B}}+e \dot{V}\right) .
$$

The series capacitance $C_{\mathrm{S}}$ arises in Eq. (16) through the time variation of $\Phi_{\mathrm{B}}$, which couples the forward and reverse biased sides of the GB. Under SBP conditions, $C_{\mathrm{L}}$ and $C_{\mathrm{R}}$ decouple as long as the measurement signal satisfies the following low frequency (LF) condition

$$
\omega \times \tau \approx 1,
$$

in which case the pinning mechanism keeps active during measurement. We refer to conditions (12) and (17) altogether as full SBP conditions. They imply that $C_{\mathrm{S}}$ disappears from (16), which simplifies to $J_{\mathrm{D}}=C_{\mathrm{R}} \times(d V / d t)=i \omega C_{\mathrm{R}} V$. The remaining term of (14) stems from expanding exp $\left(-e V / k_{\mathrm{B}} T\right)$ and $\Phi_{\mathrm{B}}$ in the capacitive part of the thermionic current $^{4} \quad J_{T}=A \times \exp \left[-\left(e \Phi_{B}+\xi\right) / k_{\mathrm{B}} T\right] \times[1-\exp$ $\left.\left(-e V / k_{\mathrm{B}} T\right)\right]$. Within full SBP conditions we neglect the $\Phi_{\mathrm{B}}$ variation, and it can be easily shown that

$$
J_{T}= \begin{cases}\left(e A / k_{\mathrm{B}} T\right) e^{-\left(\xi+e \Phi_{\mathrm{B}}^{(0)}\right) / k_{\mathrm{B}} T}\left(V_{\mathrm{dc}}+\Delta V\right) \equiv G_{0} V, & e V_{\mathrm{dc}} \ll k_{\mathrm{B}} T \text { and } e V_{0} \ll k_{\mathrm{B}} T, \\ A e^{-\left(\xi+e \Phi_{\mathrm{B}}^{(0)}\right) / k_{\mathrm{B}} T} \equiv J_{\text {sat }}, & e V_{\mathrm{dc}}>k_{\mathrm{B}} T \text { or } e V_{0}>k_{\mathrm{B}} T,\end{cases}
$$

which has no capacitive component, hence making disappear the third term in (14).

We conclude that, under full SBP regime, the series capacitance $C_{\mathrm{S}}$ and the $\omega$-dependent terms disappear from the general expression (14), which becomes simply $C(\omega)$ $=C_{\mathrm{R}}$; the $x_{\mathrm{L} 0}$ depletion edge varies negligibly and the corresponding screening charge is essentially static, adding no contribution to the total displacement current: only the reverse-biased capacitance is measured, and GB response turns out to be well described by the simple equivalent circuit in Fig. 2. If SBP conditions do not apply, then certainly $C_{\mathrm{S}}$ appears as the high frequency limit of expression (14), but Eq. (13) no longer holds: once measured, $C_{\mathrm{S}}$ could only be introduced in the generally valid Eq. (11). Now, suppose capacitance is measured in a GB under full SBP conditions: $C_{\mathrm{R}}$ is obtained, and should be introduced in Eq. (10), rewritten as

$$
1 / C_{\mathrm{R}}^{2}=2\left(\Phi_{\mathrm{B}}^{(0)}+V\right) /\left(e \varepsilon_{0} \varepsilon_{r} N_{0}\right) \equiv m\left(\Phi_{\mathrm{B}}^{0}+V\right),
$$

where we have defined the $\left(C_{\mathrm{R}}\right)^{-2}$ versus $V$ slope as $m$. Instead, if Eq. (13) is to be used, ${ }^{7-10,19}$ then the quantity $\left(1 / C_{\mathrm{R}}-1 / 2 C_{\mathrm{R}}^{(0)}\right)^{2}$ must be constructed and, deriving, we obtain

$$
\frac{d}{d V}\left(\frac{1}{C_{\mathrm{R}}}-\frac{1}{2 C_{\mathrm{R}}^{(0)}}\right)^{2}=m \times\left(1-\frac{C_{\mathrm{R}}}{2 C_{\mathrm{R}}^{(0)}}\right) \equiv m^{\prime}(V),
$$

showing that Eq. (13) does not fit a straight line, and that the measured slope of an eventual linear fit is smaller than $m$, leading to a greater donor density and a smaller barrier height. Thus, we conclude that Eq. (13) can be misleading and can lead to erroneous physical parameters.

Another important consequence of full SBP conditions is the in-phase response of the shallow donor. In the general case, the shallow charge oscillation becomes modulated with respect to the applied voltage because of the delayed variation of the barrier height. The depletion width $x_{\mathrm{R} 0} \equiv x_{\mathrm{R} 0}(t)$, given by Eq. (9), oscillates in a complex fashion, driven by the time dependency of $V$ and, also, by the more involved time dependency of $\Phi_{\mathrm{B}}$, which adds out-of-phase contributions to $x_{\mathrm{R} 0}(t)$. However, if SBP conditions can be assumed, the negligible variation of $\Phi_{\mathrm{B}}$ render these delayed contributions also negligible, and the back-and-forth oscillation of the shallow charge remains in phase with the applied voltage. We can set $V_{\mathrm{dc}}=0$ and, as the applied voltage raises from 0 to $V_{0}\left[V_{0}<V_{\Phi}\right.$ (see Eq. 12)], the depletion width increases without delay, from $x_{\mathrm{R} 0}^{(0)}$ to

$$
x_{\mathrm{R} 0}\left(V_{0}\right)=\sqrt{2 \varepsilon_{0} \varepsilon_{r}\left(\Phi_{\mathrm{B}}^{(0)}+V_{0}\right) / e N_{0}} .
$$

Hence, measuring the capacitive part of the total current that flows in response to the signal $V_{0} e^{i \omega t}$, we can extract the capacitance

$$
C_{\mathrm{R}}\left(V_{0}\right) \equiv \frac{\varepsilon_{0} \varepsilon_{r}}{x_{\mathrm{R} 0}\left(V_{0}\right)}=\sqrt{\frac{e \varepsilon_{0} \varepsilon_{r} N_{0}}{2\left(\Phi_{\mathrm{B}}^{(0)}+V_{0}\right)}} .
$$

\section{GRAIN BOUNDARY CAPACITANCE WITH DEEP DONORS}

In the general situation described by Eq. (2), continuity of the potential gives

$$
\begin{aligned}
& -\left.\Phi(x, V)\right|_{x=0} \equiv \Phi_{\mathrm{B}}(V)=\frac{e}{2 \varepsilon_{0} \varepsilon_{r}} \sum_{\alpha=0}^{d} N_{\alpha} x_{\mathrm{L} \alpha}^{2} \\
& \frac{e}{2 \varepsilon_{0} \varepsilon_{r}} \sum_{\alpha=0}^{d} N_{\alpha} x_{\mathrm{R} \alpha}^{2}=\Phi_{\mathrm{B}}(V)+V .
\end{aligned}
$$

Therefore, under SBP conditions $\left[\Phi_{\mathrm{B}}(V) \approx \Phi_{\mathrm{B}}^{(0)}\right]$, the forward-biased side of the GB remains essentially static 


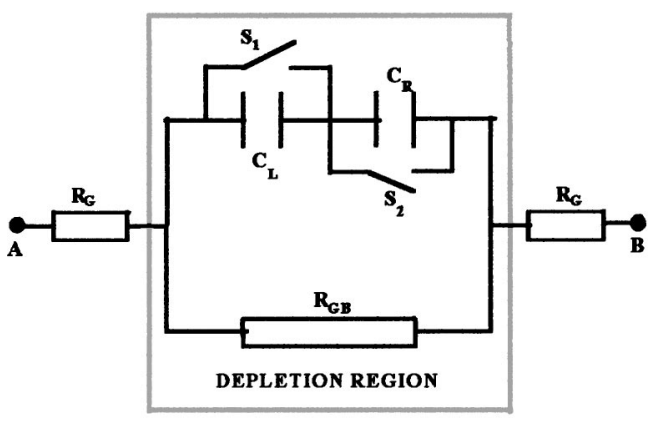

FIG. 2. Equivalent circuit for the GB response under strong barrier pinning. $R_{\mathrm{G}}$ is the bulk grain resistance and $R_{\mathrm{GB}}$ the GB resistance. When $U_{A}-U_{B}$ $<0$, then switch $S_{1}$ is closed and $S_{2}$ is open. When $U_{A}-U_{B}>0$, then $S_{1}$ is open and $S_{2}$ closed. In any case, only the capacitance of the reverse-biased depletion layer remains in the circuit.

(points $-x_{\mathrm{L} \alpha}$ remaining stationary), while the reverse-biased side behaves as a one-sided Schottky barrier with constant diffusion voltage $\mathrm{e}^{13,14,20-22}$ (points $x_{\mathrm{R} \alpha}$ moving in response to bias changes). A LF [in the sense of Eq. (17)] measurement signal $V_{0} e^{i \omega t}$ will only be responded by the deep donors of the reverse-biased side, and measured capacitance will be that of the reverse-biased depletion layer (the equivalent circuit in Fig. 2 remaining valid). The forward-biased side introduces no displacement or barrier-modulated contribution. Thus we can apply well established approximations and results, stemming from the theory of one-sided Schottky barriers where deep lying impurities are a classical subject. ${ }^{13,14,20-22}$ In particular, if $\omega$ is the measurement frequency and $\tau_{\alpha}(\alpha=1, \ldots, d)$ are the relaxation times of the deep donors, ${ }^{5}$ we assume that

$$
\begin{aligned}
& \alpha=1, \ldots, d_{\omega} \Rightarrow \omega \leqslant 2 \pi / \tau_{\alpha} \\
& \alpha=d_{\omega}+1, \ldots, d \Rightarrow \omega \gg 2 \pi / \tau_{\alpha} .
\end{aligned}
$$

In explaining this assumption, note the different footing of the shallow and deep screening charge dynamics. The former is assumed to have negligible relaxation time: out-of-phase response to the applied signal comes from the delayed dynamics of the barrier and, thus, the sole condition (12) suffices to guarantee a nondelayed shallow donor dynamics. Deep lying charge, instead, has two sources for delay: the first is still the time variation of $\Phi_{\mathrm{B}}$ and its modulation effect on the displacement currents, ${ }^{5}$ but the second is due to the finite relaxation time of deep states, that could be responding in delay even under SBP conditions. Assumption (23) means that, compared with $2 \pi / \omega$, the first $d_{\omega}$ deep donors posses negligible relaxation times, instantaneously following the test signal, while the deeper donors present effectively infinite relaxation times, remaining essentially static. Besides being well established for one-sided Schottky barriers, ${ }^{13,14}$ where it was originally introduced by Roberts and Crowell, ${ }^{22}$ this assumption seems reasonable for DSBs in ceramic semiconductors because measured relaxation times ${ }^{23}$ are separated by several decades.

When a dc bias $V_{\mathrm{dc}}$ and a measurement signal $V_{0} \exp (i \omega t)$ are applied, and (23) is assumed, the measured capacitance by unit area $C_{\mathrm{R} \omega}$ will be frequency dependent, ${ }^{13,14,21}$ even under SBP conditions:

$$
\begin{aligned}
& C_{\mathrm{R} \omega}=\varepsilon_{0} \varepsilon_{r} / X_{\mathrm{R} \omega}\left(V_{\mathrm{dc}}\right) \\
& X_{\mathrm{R} \omega}\left(V_{\mathrm{dc}}\right) \equiv\left(\sum_{\alpha=0}^{d_{\omega}} N_{\alpha} x_{\mathrm{R} \alpha}\right) /\left(\sum_{\alpha=0}^{d_{\omega}} N_{\alpha}\right),
\end{aligned}
$$

where, for fixed $V_{\mathrm{dc}}, X_{\mathrm{R} \omega}$ is an increasing function of the measurement frequency such that

$$
\lim _{\omega \rightarrow 0} X_{\mathrm{R} \omega} \equiv X_{\mathrm{R}, \mathrm{dc}} \leqslant X_{\mathrm{R} \omega} \leqslant \lim _{\omega \rightarrow \infty} X_{\mathrm{R} \omega}=x_{\mathrm{R} 0} .
$$

It is of prime importance to note that Eq. (24) can not be recast into a Mott-Schottky-like expression such as (19) because, if Eq. (2) can not be simplified to Eq. (7), then $X_{\mathrm{R} \omega}$ cannot be solved as a function of $\Phi_{\mathrm{B}}^{(0)}$ and $N_{\mathrm{D}}$ such as (9) for any frequency, not even under SBP conditions. However, it can be shown that the slope of a $\left(C_{\mathrm{R} \omega}\right)^{-2}$ versus $V$ curve is ${ }^{14}$

$$
\frac{d}{d V}\left(\frac{1}{C_{\mathrm{R} \omega}}\right)^{2}=\frac{2}{e \varepsilon_{0} \varepsilon_{r} N_{\mathrm{D}}} \frac{X_{\mathrm{R} \omega}}{X_{\mathrm{R}, \mathrm{av}}} \equiv m_{\omega},
$$

with

$$
X_{\mathrm{R}, \mathrm{av}}(V) \equiv\left(\sum_{\alpha=0}^{d} N_{\alpha} x_{\mathrm{R} \alpha}\right) /\left(\sum_{\alpha=0}^{d} N_{\alpha}\right),
$$

so the apparent donor density is given by $N_{\mathrm{D} \omega}$ $\equiv 2 /\left(e \varepsilon_{0} \varepsilon_{r} m_{\omega}\right)=\left(X_{\mathrm{R}, \text { av }} / X_{\mathrm{R} \omega}\right) N_{\mathrm{D}}$. This leads to a very important conclusion: the total donor density $N_{\mathrm{D}}$ is measured only in the low frequency limit, when all deep levels can follow the test signal $\left[d_{\omega}=d\right.$ and $X_{\mathrm{R} \omega} / X_{\mathrm{R}, \mathrm{av}}=1$ in Eq. (26)]. For any other measurement frequency, it is clear from (25) and (26) that $N_{\mathrm{D} \omega}<N_{\mathrm{D}}$.

On the other hand, the apparent barrier height given by the extrapolated intercept of the $\left(C_{\mathrm{R} \omega}\right)^{-2}$ versus $V$ curve with the voltage axis, and denoted $\Phi_{\mathrm{B} \omega}$, is known to increase with increasing frequency, ${ }^{14}$ in agreement with Ref. 5 (where the zero-bias capacitance is found to increase as the test frequency is reduced), and with the consequences on $\Phi_{\mathrm{B}}^{(0)}$ of a decreasing $N_{\mathrm{D}}$, as per Eq. (4). In this work, we take $\Phi_{\mathrm{B} \omega}$ as an (overestimated) indicative value, related with $\Phi_{\mathrm{B}}^{(0)}$ and the apparent donor density. The average barrier height can be obtained through low-field dc measurements, using (18) and ${ }^{11} \xi(T)=k_{\mathrm{B}} T \times \ln \left(N_{\mathrm{C}} / N_{0}\right)$.

\section{A. High frequency capacitance and total donor density}

If $\omega$ is so high that only the shallow donor can follow the test signal $\left(d_{\omega}=0\right)$, then the geometrical capacitance $C_{\mathrm{S}}$ $\equiv C_{\mathrm{HF}} \equiv \varepsilon_{r} \varepsilon_{0} /\left(x_{\mathrm{R} 0}+x_{\mathrm{L} 0}\right)$ is measured. ${ }^{1,5}$ But, as discussed above, an equation like (13) cannot be constructed for $C_{\mathrm{HF}}$ because an expression like (9) cannot be constructed for $x_{\mathrm{L} 0}$ and $x_{\mathrm{R} 0}$ : the voltage dependence of $\left(C_{\mathrm{HF}}\right)^{-2}$ will not fit a Mott-Schottky straight line. ${ }^{14}$ Looking for a meaning of $C_{\mathrm{HF}}$ that could justify its extended use ${ }^{6-10,19,23}$ we have to resort to the following approximation: let $\delta_{\mathrm{R} \alpha} \equiv x_{\mathrm{R} 0}-x_{\mathrm{R} \alpha}$ and $\delta_{\mathrm{L} \alpha}$ $\equiv x_{\mathrm{L} 0}-x_{\mathrm{L} \alpha}$ be the separation between the edges of the depletion layer and the edges of the ionization region for the trap $\alpha$, and suppose that 


$$
\delta_{\mathrm{R} \alpha} \ll x_{\mathrm{R} 0} ; \delta_{\mathrm{L} \alpha} \ll x_{\mathrm{L} 0} \quad \forall \alpha=1, \ldots, d
$$

Then the following rough approximation can be done in Eq. (22):

$$
\begin{aligned}
\frac{e}{2 \varepsilon_{0} \varepsilon_{r}} \sum_{\alpha=0}^{d} N_{\alpha} x_{\mathrm{R} \alpha}^{2} & =\frac{e}{2 \varepsilon_{0} \varepsilon_{r}} \sum_{\alpha=0}^{d} N_{\alpha}\left(x_{\mathrm{R} 0}-\delta_{\mathrm{R} \alpha}\right)^{2} \\
& \approx \frac{e}{2 \varepsilon_{0} \varepsilon_{r}}\left(\sum_{\alpha=0}^{d} N_{\alpha}\right) x_{\mathrm{R} 0}^{2},
\end{aligned}
$$

leading to $\left(e N_{\mathrm{D}} / 2 \varepsilon_{0} \varepsilon_{r}\right) x_{\mathrm{R} 0}^{2}=\Phi_{B}(V)+V$ and, analogously, to $\left(e N_{\mathrm{D}} / 2 \varepsilon_{0} \varepsilon_{r}\right) x_{\mathrm{L} 0}^{2}=\Phi_{\mathrm{B}}(V)$, hence recovering Eq. (9) with $N_{0}$ substituted by the total donor density $N_{\mathrm{D}}$. Under these circumstances we can reproduce the arguments leading from Eq. (9) to Eq. (13); in particular, if SBP conditions hold, we can introduce a modified Mott-Schottky equation for the high frequency capacitance:

$$
\begin{aligned}
& \left(\frac{1}{C_{\mathrm{HF}}}-\frac{1}{2 C_{\mathrm{HF}}^{(0)}}\right)^{2}=\frac{2}{e \varepsilon_{0} \varepsilon_{r} N_{\mathrm{D}}}\left(\Phi_{\mathrm{B}}^{(0)}+V\right) \\
& \left.C_{\mathrm{HF}}^{(0)} \equiv C_{\mathrm{HF}}(V)\right|_{V=0}=\frac{1}{2} \sqrt{\frac{e \varepsilon_{0} \varepsilon_{r} N_{\mathrm{D}}}{2 \Phi_{\mathrm{B}}^{(0)}}}
\end{aligned}
$$

An important conclusion follows: only under SBP conditions and with approximation (27), can a Mott-Schottky equation be written for the high frequency capacitance. In any other case, capacitance versus voltage behavior follows complicated equations that can not be put in the form (28).

Approximation (27) has been used ${ }^{20}$ in deriving analytical expressions for the current contributed by the deep traps that, in turn, have been used as a basis for characterization procedures. ${ }^{24} \mathrm{~A}$ simple test for its validity can be obtained by noting that it implies $X_{\mathrm{R}, \mathrm{av}} \approx x_{\mathrm{R} 0}$ : substituting $x_{\mathrm{R} 0}$ instead of $X_{\mathrm{R}, \mathrm{av}}$ in (26) leads to an apparent donor density that increases with frequency, and equals $N_{\mathrm{D}}$ at the high frequency limit, in contradiction with known results. ${ }^{13,14}$ Another objection against (27) is raised by the existence of deep donors with relaxation times above $1 \mathrm{~h}^{25}$ These levels lie very deep in the gap and scarcely can satisfy (27).

We conclude that, when pursuing $N_{\mathrm{D}}$ or $\Phi_{\mathrm{B}}^{(0)}$, only very low frequencies give the true donor density, and high frequency measurements are not advantageous.

\section{B. Effect of an ac bias voltage}

The above equations were obtained on the implicit assumption of a pure dc bias, i.e., an infinite waiting time between bias application and measurement, allowing all deep donors to "sense" the bias. ${ }^{13,14,21}$ If the actual measurement is performed a time $t_{m}$ after the bias application, then only those levels ${ }^{14}$ with $\tau_{\alpha} \ll t_{m}$ will contribute to (26), and the quantities $N_{\mathrm{D}}$ and $X_{\mathrm{R}, \text { av }}$ must be changed to ${ }^{14}$

$$
N_{\mathrm{D}}^{\prime} \equiv \sum_{\alpha=0}^{d_{m}} N_{\alpha}
$$

and

$$
X_{\mathrm{R}, \mathrm{av}}^{\prime}(V) \equiv\left(\sum_{\alpha=0}^{d_{m}} N_{\alpha} x_{\mathrm{R} \alpha}\right) /\left(\sum_{\alpha=0}^{d_{m}} N_{\alpha}\right),
$$

where $\alpha=0, \ldots, d_{m}$ correspond to those levels that can follow not the test signal but the bias variation. If the bias voltage and the test signal are made to vary with the same frequency $\omega$, and we denote $\alpha=0, . ., p$ the levels that can follow such signal, then the above discussion implies that Eq. (26) turns into

$$
\frac{d}{d V}\left(\frac{1}{C_{\mathrm{R} \omega}}\right)^{2}=\frac{2}{e \varepsilon_{0} \varepsilon_{r} N_{\mathrm{D} \omega}} \equiv m_{\omega} ; \quad N_{\mathrm{D} \omega} \equiv \sum_{\alpha=0}^{p} N_{\alpha} .
$$

Hence, if $C_{\mathrm{R} \omega}$ is measured in this particular situation, and used to construct a plot of $\left(C_{\mathrm{R} \omega}\right)^{-2}$ versus $V$, a straight line is obtained whose slope gives the total density of those levels that are able to follow the signals, and whose voltage-axis intercept gives an indication about equilibrium barrier height as discussed after Eq. (26). Note that, if we construct the quantity $\left[\left(C_{\mathrm{R} \omega}\right)^{-1}-\left(2 C_{\mathrm{R} \omega}^{(0)}\right)^{-1}\right]^{2}$ [with $C_{\mathrm{R} \omega}^{(0)}$ given by the extrapolated $V=0$ value of the $\left(C_{\mathrm{R} \omega}\right)^{-2}$ versus $V$ straight line], and plot it as a function of $V$, we obtain a slope

$$
\frac{d}{d V}\left(\frac{1}{C_{\mathrm{R} \omega}}-\frac{1}{2 C_{\mathrm{R} \omega}^{(0)}}\right)^{2}=m_{\omega} \times\left(1-\frac{C_{\mathrm{R} \omega}}{2 C_{\mathrm{R} \omega}^{(0)}}\right) \equiv m_{\omega}^{\prime}(V),
$$

that overestimates $N_{\mathrm{D} \omega}$ and, correspondingly, yields a value for the apparent barrier height smaller than the $\Phi_{\mathrm{B} \omega}$ that would be obtained through a plot of a plot of $\left(C_{\mathrm{R} \omega}\right)^{-2}$ versus $V$. This error is particularly deceiving now, because it compensates, in an arbitrary and uncontrolled way, the deep donor effects.

In these circumstances, and keeping in mind the discussion after Eq. (23), the arguments at the end of Sec. III B can be easily reproduced: under full SBP, the in-phase variation of $x_{\mathrm{R} \alpha}(\alpha=0, \ldots, p)$ allows $C_{\mathrm{R} \omega}\left(V_{\mathrm{dc}}\right)$ to be extracted from the capacitive component of the current that flows in response to an ac bias of amplitude $V_{0}=V_{\mathrm{dc}}$.

\section{NEW CHARACTERIZATION METHOD FOR SBP-POLYCRYSTALLINE MATERIALS}

The method we propose is based in SBP property and Eqs. (21) and (29). The usual procedure of applying a signal with $V_{0} \ll V_{\mathrm{dc}}$, therefore taking $C_{\mathrm{R} \omega}(V) \approx C_{\mathrm{R} \omega}\left(V_{\mathrm{dc}}\right)$ and sweeping $V_{\mathrm{dc}}$, is difficult to apply to devices of such technological relevance as high voltage varistors. Additionally, the size limitation of traditional impedance bridges restrains their use for testing samples that could better approach the infinite limit in which percolation theories ${ }^{26}$ are valid, and scale effects ${ }^{27}$ become important. The alternative method we propose encompasses a number of devices that make it worthy, and eludes the above-mentioned disadvantages. It consists of using the same signal $V=\Delta V \equiv V_{0} e^{i \omega t}$, both to bias the sample and to measure its capacitance. The frequency is $50-60 \mathrm{~Hz}$, which is easy to apply, lies far away from any reported relaxation, ${ }^{23,28}$ and satisfies Eq. (17) (with typical values of barrier height ${ }^{4} \Phi_{\mathrm{B}}^{(0)} \approx 0.6 \mathrm{eV}$ and GB states capture cross section ${ }^{1,4} \sigma \approx 5 \times 10^{-14} \mathrm{~cm}^{2}$ ). Smaller measurement frequencies would be desirable for higher barrier materials in order to achieve full SBP conditions. The amplitude $V_{0}$, 
ranging from $200 \mathrm{~V}_{\text {peak }}$ up to $6 \mathrm{kV}_{\text {peak }}$ (actual values used during experiment), is varied on predefined steps and, at each step satisfying $V_{0}<V_{\Phi}$ [see Eq. (12)], the capacitance $C_{\mathrm{R} \omega}\left(V_{0}\right)$ is evaluated by carefully measuring the capacitive part of the total current flowing in the external circuit. This capacitance is then used to draw a plot of $\left(C_{\mathrm{R} \omega}\right)^{-2}$ versus $V_{0}$, from which $N_{\mathrm{D} \omega}$ may be obtained and $\Phi_{\mathrm{B}}^{(0)}$ estimated. Simultaneously, the amplitude sweep is used to draw the 50 $\mathrm{Hz} I-V$ characteristic curve. Note that an additional advantage is the negligible effect of the electrodes, which are usually conflictive when measuring thin samples but are totally negligible when the measured sample is more than two orders of magnitude thicker than the electrode.

\section{EXPERIMENTAL PROCEDURE}

Samples for the above described method were highvoltage (rated $\left.{ }^{2} 3 \mathrm{kV}_{\mathrm{rms}}\right)$, cylindrically shaped $(\varnothing 4.2 \mathrm{~cm}$ and $2.2-2.7 \mathrm{~cm}$ height) $\mathrm{ZnO}$ varistors, with arc sprayed $\mathrm{Al}$ electrodes. Average grain size was known to be $15 \mu \mathrm{m}$. The active cross-section area for ac current flow has been taken to be equal to the electrode area: studies about current concentration ${ }^{2,26,27}$ focus only on the dc transport properties and yield no definite criteria about the influence of electrical/ structural disorder on the ac properties. The method was illustrated through a specific problem: that of relating the physical parameters of a device, before and after severe electrical degradation. A sample was measured, then heavily degraded with $100 \mathrm{kA}$ under-damped current pulses, and measured again.

In order to check the presence of SBP conditions we note that, according to (18), current is an exponentially sensitive detector of changes in $\Phi_{\mathrm{B}}$. Hence, several discs were sliced from the samples (typically $\varnothing 12 \mathrm{~mm}$ and $0.5 \mathrm{~mm}$ height), and used to verify SBP through dc-conductivity measurements. The discs were also used to check the presence of very deep donors (recording the absorption current due to a dc voltage, hence observing long-time relaxation phenomena), and to obtain alternative measurements of the barrier height, through the method explained after (26). All these experiments were performed on a KEITHLEY 6517A electrometer.

\section{RESULTS}

Figure 3 shows the dc measurements performed on small discs. The appearance of a subohmic region above the linear $e V_{\mathrm{dc}} \ll k_{\mathrm{B}} T$ regime, is to be considered an indication of negligible changes in the barrier height. ${ }^{5}$ Figure 4 shows the time-domain dielectric response of a small disc (for various dc applied voltages): relaxation phenomena delaying up to half an hour were observed, hence confirming the presence of very deep donors and the general inadequacy of hypothesis (27).

Figure 5 shows the raw output of the method: total 50 $\mathrm{Hz}$ current, in-phase component and nonlinearity coefficient $\alpha .^{2}$ SBP property is assumed to hold during the lowervoltage portions of these figures: barrier change within the voltage cycle introduces nonlinear phenomena, easily detected through the nonlinearity coefficient and the harmonic

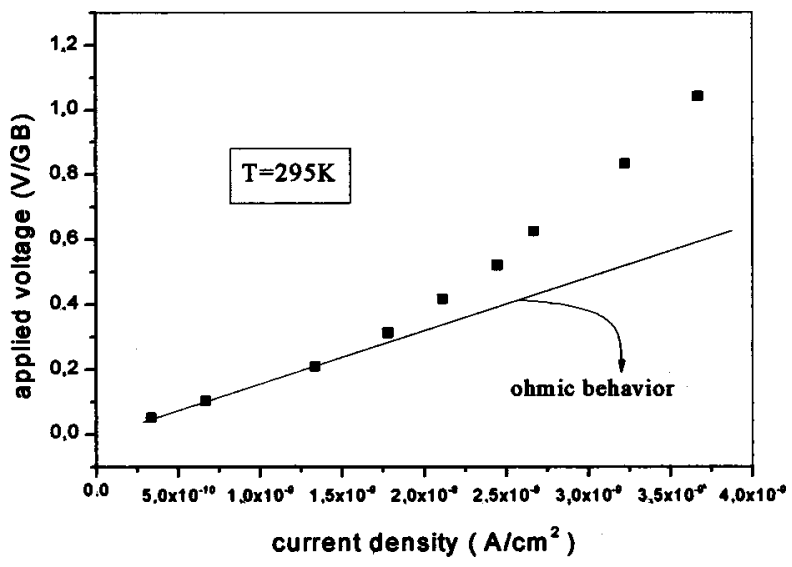

FIG. 3. A typical low-field, dc response of a disc sliced from a high voltage sample. The relevant feature of the figure is the clear appearance of a subohmic behavior due to barrier pinning.

distortion of the current signal. Figure 6 shows the corresponding Mott-Schottky plot: the left $y$-axis represents the measured $\left(C_{\mathrm{R} \omega}\right)^{-2}$, while the right $y$-axis represents the modified quantity $\left[\left(C_{\mathrm{R} \omega}\right)^{-1}-\left(2 C_{\mathrm{R} \omega}^{(0)}\right)^{-1}\right]^{2}$. The overestimation of $N_{\mathrm{D} \omega}$ [Eq. (30)] is apparent: from the left $y$-axis, we deduce $N_{\mathrm{D} \omega} \approx 8.6 \times 10^{16} \mathrm{~cm}^{-3}$ and $\Phi_{\mathrm{B} \omega} \approx 1.5 \mathrm{~V}$; from the right $y$-axis we deduce $N_{\mathrm{D} \omega} \approx 1.2 \times 10^{17} \mathrm{~cm}^{-3}$ and $\Phi_{\mathrm{B} \omega}$ $\approx 0.6 \mathrm{~V}$. According to theoretical considerations, ${ }^{1,4}$ typical values of the total donor density lie in the range $N_{\mathrm{D}} \approx 10^{17}$ $-10^{18} \mathrm{~cm}^{-3}$. Therefore, the arbitrary compensation introduced in the plot based on the quantity $\left[\left(C_{\mathrm{R} \omega}\right)^{-1}\right.$ $\left.-\left(2 C_{\mathrm{R} \omega}^{(0)}\right)^{-1}\right]^{2}$ is clearly illustrated: the overestimated value of $N_{\mathrm{D} \omega}$ lies within the expected range for $N_{\mathrm{D}}$, and the apparent barrier height yields realistic values ${ }^{1,2}$ (the $\Phi_{\mathrm{B}}^{(0)}$ value derived from $I-V$ measurements ${ }^{29,30}$ is actually $\sim 0.6 \mathrm{~V}$ ), but this is an spurious and uncontrolled effect. Deep donors do exist and the measured values for the donor density $N_{\mathrm{D} \omega}$ and barrier height $\Phi_{\mathrm{B} \omega}$ must be lower and higher, respectively, than the real values, as explained after Eq. (30). Instead, the $N_{\mathrm{D} \omega}$ obtained from the standard $\left(C_{\mathrm{R} \omega}\right)^{-2}$ versus $V$ plot through Eq. (29), gives the exact density of the donor levels that follow the signal, hence reflecting the physics behind.

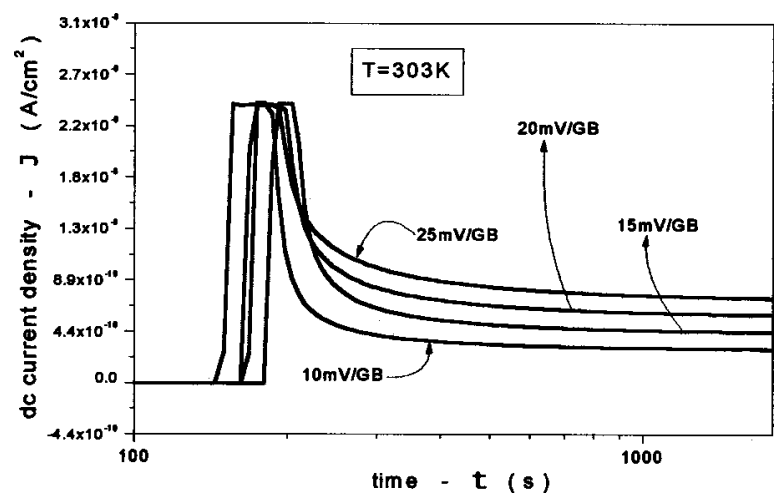

FIG. 4. Typical time domain dielectric response measured on a disc sliced from a high voltage sample. The figure shows the current flowing in response to a dc voltage of the indicated value, which was applied and sustained for at least $30 \mathrm{~min}$. 


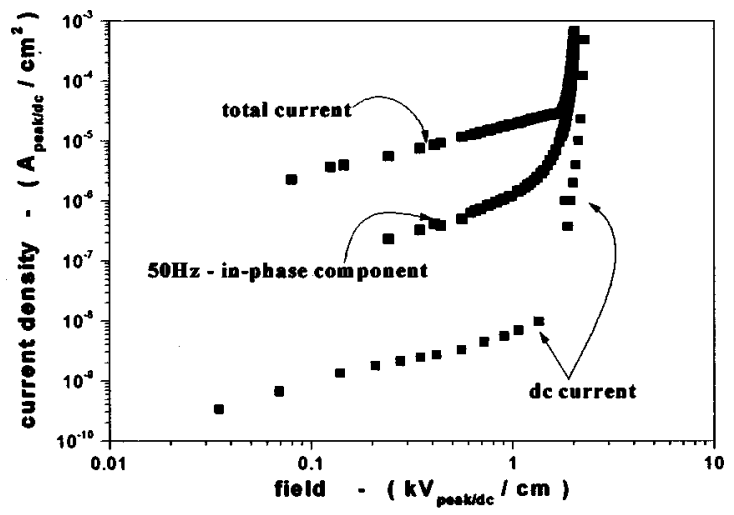

(a)

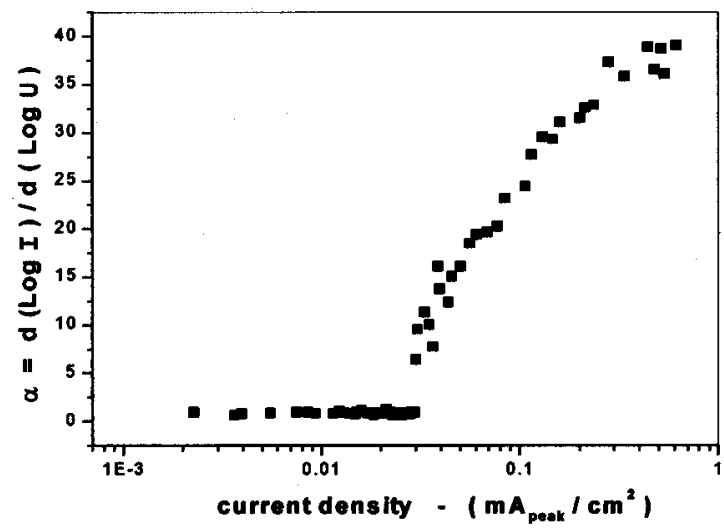

(b)

FIG. 5. An example of the output of the presented method. The total $50 \mathrm{~Hz}$ current contains both capacitive and resistive contributions. For comparison purposes, we also show in (a) the true dc $I-V$ characteristic obtained from a disc sliced from a high voltage sample.

Figures $7(\mathrm{a})-7(\mathrm{c})$ show comparative $\alpha-I, I-V$, and $C-V$ measurements from a sample, before and after degradation, as explained above. The physical parameters deduced from the $C-V$ plot are $N_{\mathrm{D} \omega} \approx 8.6 \times 10^{16} \mathrm{~cm}^{-3}, \Phi_{\mathrm{B} \omega} \approx 1.5 \mathrm{~V}$ before degradation, and $N_{\mathrm{D} \omega} \approx 4.8 \times 10^{16} \mathrm{~cm}^{-3}, \quad \Phi_{\mathrm{B} \omega}$ $\approx 0.7 \mathrm{~V}$ after degradation. We observe several changes. First, a drastically reduced nonlinearity that points to a reduction in $N_{\mathrm{D}}$ (apparent from $C-V$ characterization), below the threshold level that triggers minority-carrier production; ${ }^{1,31}$ thus,

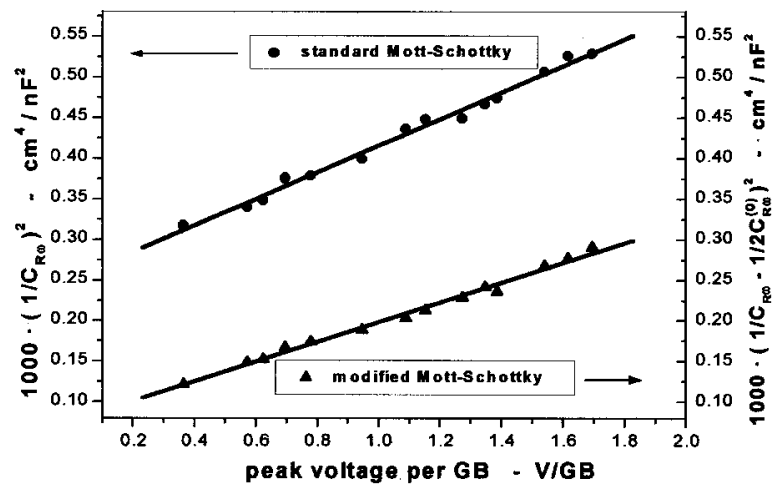

FIG. 6. A $C^{-2}-V$ curve, presented as per the standard and modified MottSchottky equations.

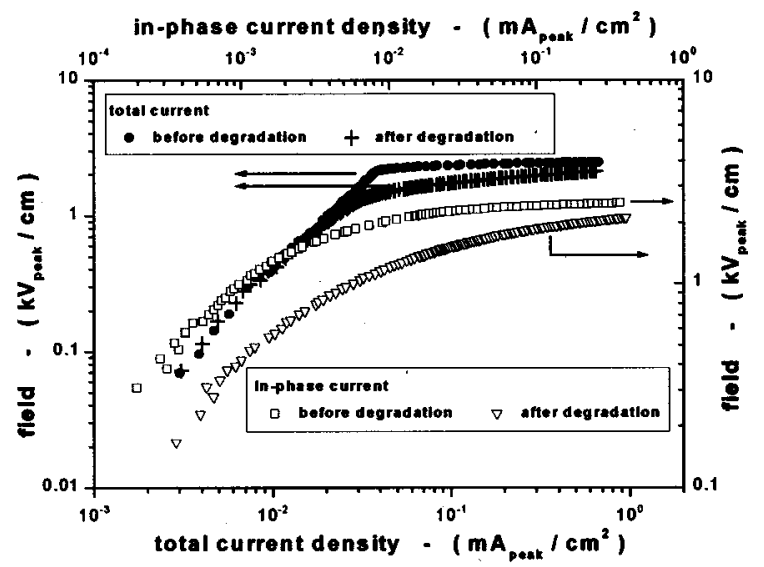

(a)

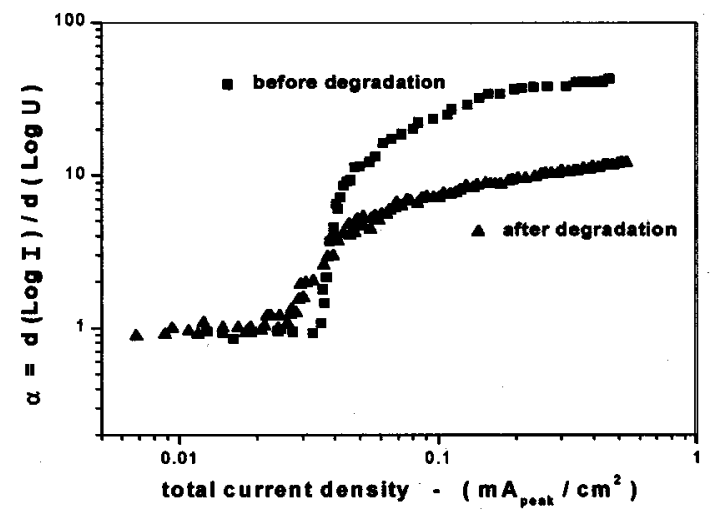

(b)

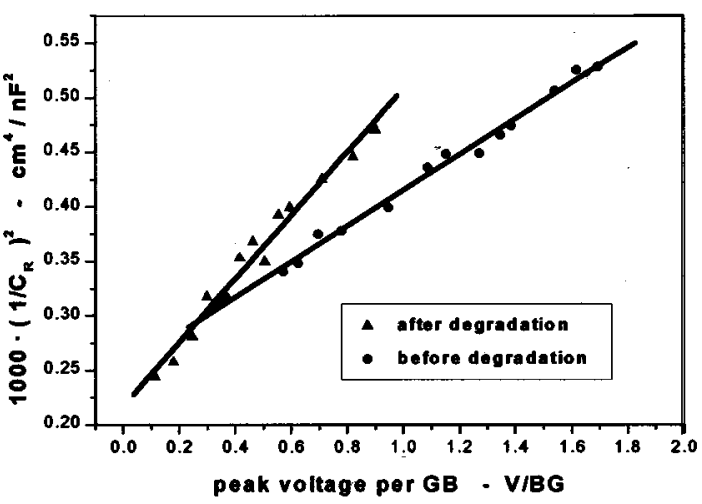

(c)

FIG. 7. Sequence of changes suffered by a sample after severe electrical degradation. The physical parameters deduced from the $C^{-2}-V$ plot are $N_{\mathrm{D} \omega} \approx 8.6 \times 10^{16} \mathrm{~cm}^{-3}, \quad \Phi_{\mathrm{B} \omega} \approx 1.5 \mathrm{~V}$ before degradation and $N_{\mathrm{D} \omega} \approx 4.8$ $\times 10^{16} \mathrm{~cm}^{-3}, \Phi_{\mathrm{B} \omega} \approx 0.7 \mathrm{~V}$ after degradation.

degradation seems to cause a transition (from a high-GB field, hot-electrons model $^{31}$ to a thermionic-emission-only model) in the nature of the conduction mechanism. Second, a shift in the onset of nonlinear phenomena towards the lowfield region, suggesting that GB states have been lost with degradation; the interface fills faster, the barrier begins to vary at much lower fields and the regime of validity of SBP conditions is reduced. Third, an increase in low-field currents, pointing to a reduction of the barrier height, also indicated by the decrease of $\Phi_{\mathrm{B} \omega}$ in the $C-V$ plot. Note that, 
from Eq. (4), the decrease in $N_{\mathrm{D}}$ should produce an increase in $\Phi_{\mathrm{B}}^{(0)}$, unless accompanied by the detected reduction in the GB trapped charge. The $\Phi_{\mathrm{B}}^{(0)}$ values $(\sim 0.6 \mathrm{~V}$ before and $\sim 0.3 \mathrm{~V}$ after degradation) obtained from small slabs, support the barrier reduction $\left(\mathrm{see}^{29,30}\right.$ for the relation between $I-V$ and $C-V$ barrier measurements).

\section{DISCUSSION}

A property called strong barrier pinning has been defined, and shown to underlie the use of Mott-Schottky behavior for physical characterization of polycrystalline semiconductors. The consequences of SBP on capacitance versus voltage behavior, both with and without deep donors, have been explored and the following casuistry has been established:

(1) Under full SBP regime and with negligible deep donor contribution, measured capacitance shows no dispersion, and corresponds to that of the reverse-biased side of the depletion layer. The standard Mott-Schottky plots [Eq. (19)] yield straight lines and give the values for barrier height and donor density. The modified MottSchottky plots [Eq. (13)] can be misleading and can give higher donor densities and lower barrier heights. The shallow screening charge and the reverse-biased depletion edge oscillate in phase with the measuring signal, permitting the capacitance at voltage $V_{\mathrm{dc}}$ to be measured directly through a signal with amplitude $V_{0}=V_{\mathrm{dc}}$.

(2) Under SBP regime and with deep donors, a MottSchottky-like equation is no longer available. MottSchottky behavior could be recovered with high frequency measurements, under an approximation that seems unjustified, according to experimental facts. Any attempt to use a modified Mott-Schottky equation [Eq. (13) with $N_{\mathrm{D}}$ instead of $N_{0}$ ] is particularly deceiving, because it introduces an arbitrary correction that partially compensates the deep donor contribution and obscures the underlying phenomena. The slope of a $C^{-2}$ versus $V$ plot yields the total donor density at the low-frequency limit, and high frequency measurements present no special advantage. When the applied bias is transformed to ac, and made to coincide with the measurement signal, a low-frequency $C^{-2}$ versus $V$ plot can be obtained by sweeping the ac signal amplitude (as in (1) above), and its slope gives the exact density of donors that follow the signal.

(3) When an SBP regime does not exist (or the applied voltage lies beyond the SBP threshold) then, MottSchottky behavior does not exist. Measured capacitance is strongly dispersive and mixes the reverse- and forward-biased sides. Its high frequency limit yields the total, geometrical series capacitance of the forward and reverse biased sides of the depletion region. But this high-frequency capacitance can not be introduced in a Mott-Schottky equation, neither standard nor modified, not even when only shallow donors are present, because its voltage variation strongly depends upon barrier dynamics.

\section{CONCLUSION}

Necessary conditions for Mott-Schottky behavior in double Schottky barriers, with and without deep donors, have been established. Under these conditions, capacitance behavior and $C-V$ interpretation schemes (beyond pure Mott-Schottky behavior), have been discussed. A widely applied Mott-Schottky-like equation [Eq. (13)] has been shown to be misleading when deep donors are not negligible, and a new characterization method has been proposed, well suited for ceramic varistors and directly applicable to highvoltage samples. It allows comparative measurements of nonlinearity, $I-V$ characteristic, donor density and barrier height to be easily done, hence being of technological relevance.

\section{ACKNOWLEDGMENTS}

The authors acknowledge S.A. INAEL and S.A. IKV for their interest in our work and the kind permission to use the High Voltage Laboratory. This work has been supported by the CICYT under Projects COO1999-AX012 and MAT20011862-C02.

${ }^{1}$ G. Blatter and F. Greuter, Semicond. Sci. Technol. 5, 111 (1990).

${ }^{2}$ D. R. Clarke, J. Am. Chem. Soc. 82, 485 (1999).

${ }^{3}$ G. E. Pike and C. H. Seager, J. Appl. Phys. 50, 3414 (1979).

${ }^{4}$ G. E. Pike, Phys. Rev. B 30, 795 (1984).

${ }^{5}$ G. Blatter and F. Greuter, Phys. Rev. B 33, 3952 (1986).

${ }^{6}$ P. R. Bueno, M. R. de Cassia-Santos, E. R. Leite, E. Longo, J. Bisquert, G. Garcia-Belmonte, and F. Fabregat-Santiago, J. Appl. Phys. 88, 6545 (2000).

${ }^{7}$ J. Fan and R. Freer, J. Appl. Phys. 77, 4795 (1995).

${ }^{8}$ S.-N. Bai and T.-Y. Tseng, J. Appl. Phys. 74, 695 (1993).

${ }^{9}$ Y.-S. Lee and T.-Y. Tseng, J. Mater. Sci.: Mater. Electron. 9, 65 (1998).

${ }^{10}$ E. D. Kim, M. H. Oh, and Ch. H. Kim, J. Mater. Sci. 21, 65 (1998).

${ }^{11}$ S. M. Sze, Physics of Semiconductor Devices, 2nd Ed. (WileyInterscience, New York, 1981).

${ }^{12}$ K. Mukae, K. Tsuda, and I. Nagasawa, J. Appl. Phys. 50, 4475 (1979).

${ }^{13}$ J.-J. Shiau and R. H. Bube, Solid-State Electron. 29, 1153 (1986).

${ }^{14}$ J.-J. Shiau, A. L. Fahrenbruch, and R. H. Bube, J. Appl. Phys. 59, 2879 (1986)

${ }^{15}$ G. E. Pike, Phys. Rev. B 30, 3274 (1984).

${ }^{16}$ G. C. McGonigal, D. J. Thomson, J. G. Shaw, and H. C. Card, Phys. Rev. B 28, 5908 (1983).

${ }^{17}$ M. G. Spencer, W. J. Schaff, and D. K. Wagner, J. Appl. Phys. 54, 1429 (1983).

${ }^{18}$ W. E. Taylor, N. H. Odell, and H. Y. Fan, Phys. Rev. 88, 867 (1952).

${ }^{19}$ C.-W. Nahm and B.-C. Shin, J. Mater. Sci.: Mater. Electron. 13, 111 (2002).

${ }^{20}$ G. Vincent, D. Bois, and P. Pinard, J. Appl. Phys. 46, 5173 (1975).

${ }^{21}$ L. C. Kimerling, J. Appl. Phys. 45, 1839 (1974).

${ }^{22}$ G. I. Roberts and C. R. Crowell, J. Appl. Phys. 41, 1767 (1970).

${ }^{23}$ M. A. Alim, M. A. Seitz, and R. W. Hirthe, J. Appl. Phys. 63, 2337 (1988).

${ }^{24}$ J. F. Cordaro, Y. Shim, and J. E. May, J. Appl. Phys. 60, 4186 (1986).

${ }^{25}$ L. M. Levinson and H. R. Philipp, J. Appl. Phys. 47, 3177 (1976).

${ }^{26}$ M. Bartkowiak and G. D. Mahan, Phys. Rev. B 51, 10825 (1995).

${ }^{27}$ H. Wang, M. Bartkowiak, F. A. Modine, R. B. Dinwiddie, L. A. Boatner, and G. D. Mahan, J. Am. Chem. Soc. 81, 2013 (1998).

${ }^{28}$ T. Asokan and R. Freer, J. Eur. Ceram. Soc. 11, 545 (1993).

${ }^{29}$ G. D. Mahan, J. Appl. Phys. 55, 980 (1984).

${ }^{30}$ J. H. Werner and H. H. Güttler, J. Appl. Phys. 69, 1522 (1991).

${ }^{31}$ G. Blatter and D. Baeriswyl, Phys. Rev. B 36, 6446 (1987). 